\title{
Progression of asymptomatic bifascicular block to complete heart block during upper gastrointestinal endoscopy with propofol sedation
}

\author{
Steven T. Morozowich, DO $\cdot$ Stuart B. Saslow, MD
}

Received: 11 August 2008/Revised: 24 September 2008/ Accepted: 26 September 2008/Published online: 19 December 2008 (c) Canadian Anesthesiologists' Society 2008

To the Editor,

The natural progression of asymptomatic bifascicular block to complete heart block (CHB) in elderly patients occurs with a frequency of approximately $1 \%$ per year, and the risk of developing $\mathrm{CHB}$ in this population under general anesthesia has not been shown to be accelerated, thus preoperative permanent pacemaker placement is not recommended. Recently, an 87-year-old female with asymptomatic bifascicular block developed ventricular asystole and CHB during upper gastrointestinal (GI) endoscopy under propofol sedation at our institution. Her medical history was significant for dysphagia, weight loss, hypertension, and a $60 \%$ right coronary artery stenosis. Previous nuclear medicine stress testing revealed normal left ventricular systolic function and no ischemia. The preprocedure electrocardiogram demonstrated normal sinus rhythm with right bundle branch block and left anterior fascicular block (bifascicular block). She was not taking any medications with atrioventricular nodal-blocking activity and routine laboratory screening tests were within normal limits. The patient denied a history of syncope or other symptoms suggestive of CHB. Her blood pressure was $171 / 83 \mathrm{mmHg}$ and heart rate was 83 beats $\mathrm{min}^{-1}$. In the endoscopy suite, standard American Society of Anesthesiologists monitors were applied. Oxygen $61 \mathrm{~min}^{-1}$ was administered via facemask and the patient was positioned left lateral decubitus. During the 13-min endoscopy a registered nurse titrated $80 \mathrm{mg}$ of propofol $i v$, as directed by the gastroenterologist performing the procedure. Patient response to sedation was maintained at purposely responsive to either verbal or tactile physical stimuli throughout

S. T. Morozowich, DO $(\bowtie) \cdot$ S. B. Saslow, MD Mercy Regional Medical Center, Durango, USA e-mail: stevenmorozowich@mercydurango.org the brief procedure. Her heart rate ranged from 77 to 90 beats $\min ^{-1}$, there was no hypotension, and no ST segment changes. Oxygen saturation was maintained at $98-100 \%$ and ventilation by observation and facemask capnography revealed no apparent apnea. At the end of the procedure a midesophageal biopsy and three sequential esophageal dilations $(50,52$, and 54-French) were done. Immediately following these interventions, the patient developed ventricular asystole and CHB (Fig. 1) lasting approximately $30 \mathrm{~s}$. Upon recognition of the arrhythmia, the patient was positioned supine where the electrocardiogram then revealed spontaneous return to normal sinus rhythm with heart rate 75 beats $\min ^{-1}$ and blood pressure 159/ $72 \mathrm{mmHg}$. No pharmacological interventions were required and there were no recurrent arrhythmias. Cardiology consultation recommended placement of a permanent pacemaker prior to discharge, which was done uneventfully.

Propofol has been associated with bradycardia, heart block, and asystole, ${ }^{1}$ but because of its favourable pharmacologic profile it is being used routinely for GI endoscopy. Vagal stimulation during GI endoscopy is common, but there are few published reports of cardiovascular complications. One report describes a $1.7 \mathrm{~s}$ ventricular pause ${ }^{2}$ and another report presents a case of ventricular asystole related to electrocautery use $^{3}$ during GI endoscopy. Considering our patient's comorbidities, any development of hypotension, an exacerbation of pulmonary hypertension (e.g., hypoxia and/or hypercarbia) and/or increased myocardial demand would compromise coronary perfusion to the physiologic cardiac pacemakers. Since no hypotension or hypoxia occurred during the procedure and no opioids were given, it is plausible that the combination of underlying conduction system disease in the setting of propofol use and vagal stimulation provided the necessary 
Fig. 1 Electrocardiogram rhythm strip (lead II) recorded during the patient's progression to ventricular asystole and complete heart block. a Demonstrates normal sinus rhythm with first degree atrioventricular block, followed by ventricular asystole with an atrial rate of approximately 50 beats $\min ^{-1}$. b Subsequently demonstrates a ventricular escape beat characteristic of complete heart block.

Additional tracings could not be obtained due to technical factors
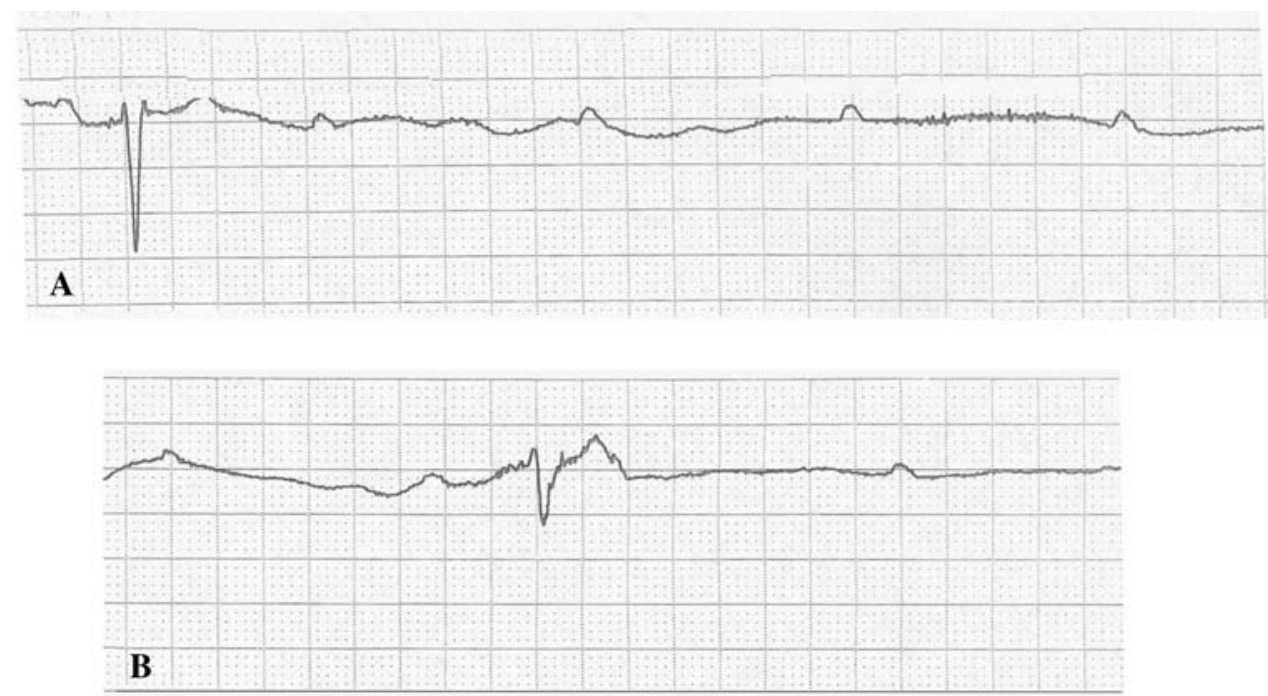

synergy to produce ventricular asystole and CHB in this patient. Asymptomatic bifascicular block in patients with advanced age and cardiac comorbidities, similar to the patient described here, has been reported as a risk factor for the development of CHB under general anesthesia, ${ }^{4,5}$ and a call by one author to recommend cardiology consultation for preoperative prophylactic permanent pacemaker placement in this population. ${ }^{4}$ Although anticholinergic agents can prevent and treat vagally mediated arrhythmias in healthy patients during surgery under general anesthesia, ${ }^{6}$ the prophylactic efficacy of these agents given prior to GI endoscopy in patients with underlying conduction system disease to prevent CHB is unknown, and the risks of these agents (e.g., disturbing the delicate balance between myocardial oxygen delivery and demand and central nervous system effects in the elderly patient) must be taken into consideration on a case-by-case basis. As this experience demonstrates, the combination of GI endoscopy, propofol sedation, and asymptomatic bifascicular block in the elderly comorbid patient may be risk factors for developing CHB, thus anesthesiologist consultation and the placement of preprocedure transcutaneous pacemaker pads for these and related procedures should be considered.
Funding sources None.

Conflicts of interest None declared.

\section{References}

1. Tramer MR, Moore RA, McQuay HJ. Propofol and bradycardia: causation, frequency and severity. Br J Anaesth 1997; 78: 642-51.

2. Mathew PK, Ona FV, Damevski K, Wallace WA. Arrhythmias during upper gastrointestinal endoscopy. Angiology 1979; 30: 834-40.

3. Rubeiz GJ, Tobi M, Meissner MD. Ventricular asystole during upper gastrointestinal endoscopic electrocoagulation. Gastrointest Endosc 1995; 41: 261-3.

4. Boezaart AP, Clinton $C W$, Stanley A. Pre-operative prophylactic transvenous cardiac pacing for bifascicular heart block. S Afr J Surg. 1989; 27: 103-5.

5. Gauss A, Hubner C, Radermacher P, Georgieff M, Schutz W. Perioperative risk of bradyarrhythmias in patients with asymptomatic chronic bifascicular block or left bundle branch block: does an additional first-degree atrioventricular block make any difference? Anesthesiology 1998; 88: 679-87.

6. Deschamps A. Lumbo-sacral spine surgery and severe bradycardia (Letter). Can J Anaesth 2004; 51: 277. 